\title{
Triple Tangent Flank Milling of Ruled Surfaces
}

\author{
Cornelia Menzel $^{1}$, Sanjeev Bedi ${ }^{2}$, Stephen Mann ${ }^{3}$ \\ University of Waterloo, Waterloo, Ontario, Canada. N2L 3G1. (519) 888-4567, \\ FAX (519) 888-6197
}

\begin{abstract}
This paper presents a positioning strategy for flank milling ruled surfaces. It is a modification of a positioning method developed by Bedi et al. [1]. A cylindrical cutting tool is initially positioned tangential to the two boundary curves on a ruled surface. Optimization is used to move these tangential points to different curves on the ruled surface to reduce the error. A second optimization step is used to additionally make the tool tangent to a rule line, further reducing the error and resulting in a tool position where the tool is positioned tangential to two guiding rails and one rule line. The resulting surface has $88 \%$ less under cutting than the Bedi et al. method.
\end{abstract}

Key words: 5-axis machining, flank milling, machine simulation, tool path generation

\section{Introduction}

In this paper, we present a method for flank milling of ruled surfaces. In many ways flank milling provides a greater challenge than point milling of complex surfaces. However, there are many advantages to flank milling. Since the whole length of the cutter flank is involved in the cutting process, the metal removal rate can be high. Furthermore, no scallops are left behind in single pass flank milling so that less surface finishing work is required. Therefore, flank milling is especially well suited for applications like impellers and turbine blades. Since for these parts, performance is particularly dependent on the surface design, it is important

1 Email: Cornelia.Menzel@gmx.de, Exchange student, Technische Universität Braunschweig, Braunschweig, Germany

2 Email: sbedi@uwaterloo.ca, Professor, Mechanical Engineering

3 Email: smann@cgl.uwaterloo.ca, Associate Professor, Computer Science 
to develop positioning strategies for flank milling that lead to a minimum error between the designed surface and machined surface.

In comparison to other areas of research, relatively little has been published in the area of flank milling [1-7]. Studies in this area focus mainly on the machining of ruled surfaces. Several positioning strategies for five-axis machining of ruled surfaces have been developed to minimize deviations between the actual machined surface and the designed ruled surface.

Bohez et al. [2] developed an algorithm that positions the tool tangent to a point $P$ on the rule line. They require that the angles between the surface normal at point $P$ and the surface normals on the two guiding rails are equal. The resulting undercut can be reduced further by moving the tool axis away from the surface along the normal at point $P$ until the tool is approximately tangent to the two guiding rails. However, this approximation is only appropriate for low curvature guiding rails. The method produces a maximum overcut in the middle area of the ruled surface. By introducing multiple pass machining the overcut was further reduced.

Liu [3] presents two methods for generating cutter location data. The single point offset method (SPO) sets the tool axis collinear with the rule line and through a point offset in the direction of the surface normal at the mid-curve. Liu's second method is a double point offset (DPO) strategy. The general idea is to offset two points on the rule line at parametric values of 0.25 and 0.75 . The points are offset along the surface normals by the cutter radius respectively. The two offset points are then used to define the tool axis orientation. The DPO method introduces significant undercut error along the mid-curve of the ruled surface.

A recent approach to machining a ruled surface was developed by Tönshoff et al. [6]. In their work, the desired surface is offset by the tool radius. The second step is to fit a ruled surface to the offset surface. The straight lines of the ruled surface are the tool axes for the tool path. This method leads to an unevenly distributed and uncontrollable error over the entire surface.

Redonnet et al. [4] suggest positioning a cylindrical cutting tool tangential to the ruled surface at three points by slightly changing the angle between the tool axis and the rule line. They developed a system of seven transcendental equations that must be solved simultaneously to obtain each tool position. The system of equations limits the robustness and relatively long computation times are required.

Tsay and Her [8] give a different approach to optimizing the tool position for flank milling of ruled surfaces with a cylindrical cutter. They analyze the error in planes perpendicular to the rule line, and then use statistical analysis to derive equations to minimize this error.

Bedi et al. [1] developed a strategy to roll a cylindrical cutting tool along two guiding rails. The tool remains tangent to the guiding rails at all times. The contact points on the two guiding curves are at the same parameter value. To machine ruled surfaces, they use the boundary curves of the ruled surface as guiding rails. Higher order surfaces can be machined 
similarly. This method is easy to implement, robust and fast to compute, since only two transcendental equations need to be solved numerically. Additionally, this method leads to a predictable deviation. Nonetheless, if this strategy is used to machine a ruled surface, the cutting tool will be tangential to the designed surface only at two points for any given tool position. In the entire area between the boundary curves, severe undercutting will occur; i.e., the tool will remove material beyond the designed surface. The maximum deviation will lie at the mid-curve of the surface.

Since these strategies are computationally expensive or lead to uncontrollable or large deviations, a better positioning method is needed. With Bedi et al.'s strategy serving as a basis, we developed an optimized method that leads to a significant reduction in deviation compared to the previous strategy.

In our new strategy, the toolpath consists of numerous tool positions each of which places the tool as closely as possible to a different rule line on the surface. The method of placing a tool relative to two guiding rails is based on Bedi et al.'s rolling cylinder method. Initially the tool is placed tangential to the surface at the ends of the rule lines, just as in the rolling cylinder method. In the next step, the positioning points are moved inwards along the rule lines until the deviation between the rule line and the cutting tool is minimized for this search-direction. In the final step the positioning points are moved sideways until the deviation between the rule line and cylindrical tool is further minimized. The strategy results in a reduction of more than $88 \%$ in undercutting. The rolling cylinder method developed by Bedi et al. is presented first for completeness. The details of the optimized strategy are given in Section 2.2.

Our method is similar to Redonnet et al.'s method [4] in that both methods place the tool tangent to the rule line and both rails. The primary difference is that while Redonnet et al. solve a system of seven transcendental equations, our method requires solving a system of only two transcendental equations.

\section{Implementation}

\subsection{Mathematical background}

Bedi et al.'s tangential positioning strategy places the cutting tool tangential to the top and bottom curve of the ruled surface at equal parametric values $u$. The positioning strategy computes two points $\left(v_{1}\right.$ and $\left.v_{2}\right)$ on the tool axis that fix the tool orientation. The first step is to establish the Frenet frames for both guiding curves. The Frenet frame for $T(u)$ is defined by the tangent $T_{t}(u)$, the main normal $T_{m}(u)$ and the binormal $T_{b}(u)$. Similarly, $B_{t}(u), B_{m}(u)$ and $B_{b}(u)$ establish the Frenet frame at $B(u)$.

To position a cylindrical tool tangential to curve $T$, the tool axis must pass through a plane perpendicular to $T_{t}$. The resulting intersection point $v_{1}$ lies a distance $R_{\text {tool }}$ from curve 


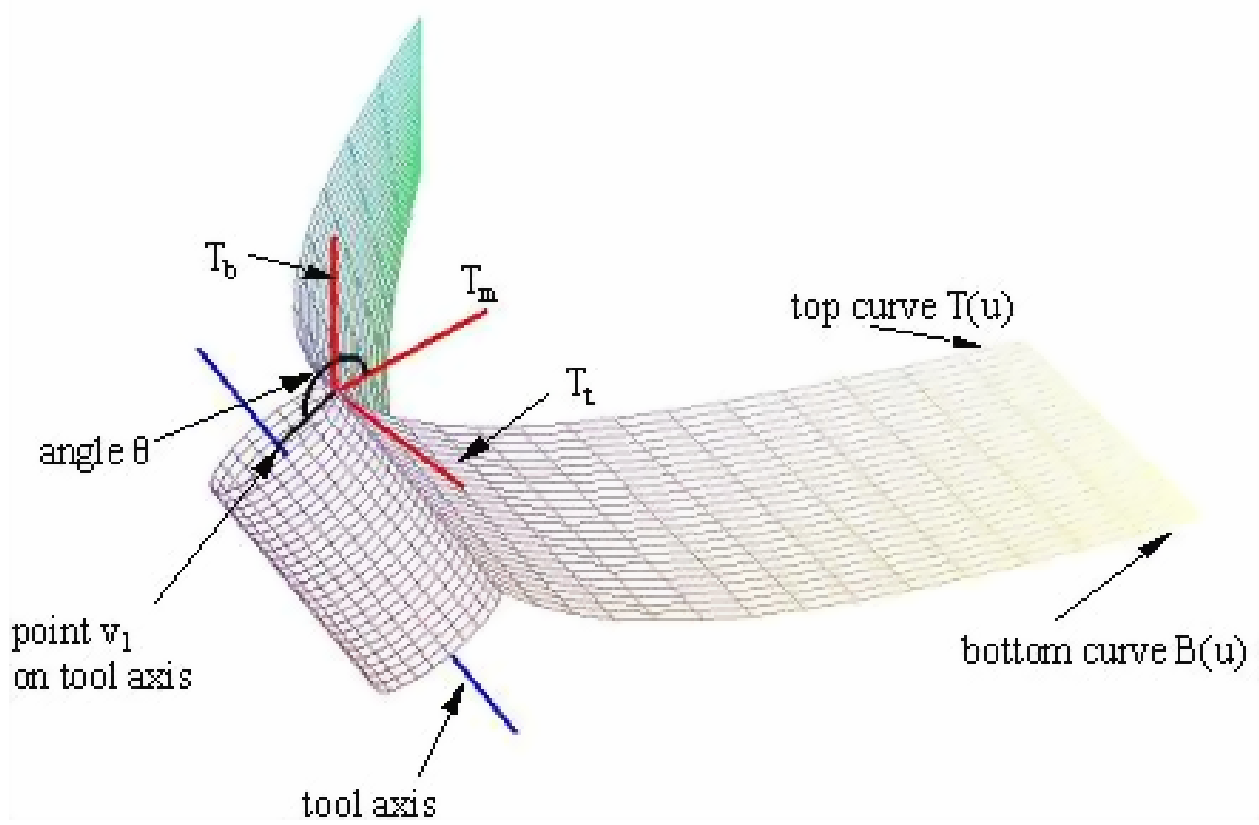

Fig. 1. Cylindrical tool tangential to two guiding curves

$T$, where $R_{\text {tool }}$ is the radius of the cylinder. The vector from $T(u)$ to $v_{1}$ lies in the plane described by $T_{b}$ and $T_{m}$ at an angle $\theta$ towards $T_{m}$. Figure 1 shows the Frenet frame for the top curve as well as the positioning angle $\theta$ and point $v_{1}$ on the tool axis. Similarly, a second point $v_{2}$ on the tool axis lies in the $B_{b}-B_{m}$ plane at distance $R_{\text {tool }}$ from curve $B(u)$. The angle between $B_{m}$ and the vector from $B(u)$ to $v_{2}$ is defined as $\beta$. With $v_{1}$ and $v_{2}$ defined by $\theta$ and $\beta$, the tool axis can be set as $v_{1}-v_{2}$. Equations (1) and (2) developed by Bedi et al. show this relationship:

$$
\begin{aligned}
& v_{1}-T(u)=R_{\text {tool }} \cdot \cos (\theta) \cdot T_{m}(u)+R_{\text {tool }} \cdot \sin (\theta) \cdot T_{b}(u) \\
& v_{2}-B(u)=R_{\text {tool }} \cdot \cos (\beta) \cdot B_{m}(u)+R_{\text {tool }} \cdot \sin (\beta) \cdot B_{b}(u)
\end{aligned}
$$

For cylindrical tools, the axis $\left(v_{1}-v_{2}\right)$ must be perpendicular to the two vectors $\left(v_{1}-T\right)$ and $\left(v_{2}-B\right)$. Thus, the dot product must equal zero:

$$
\begin{array}{r}
\left(v_{1}-v_{2}\right) \cdot\left(\cos (\theta) \cdot T_{m}+\sin (\theta) \cdot T_{b}\right)=0 \\
\left(v_{1}-v_{2}\right) \cdot\left(\cos (\beta) \cdot B_{m}+\sin (\beta) \cdot B_{b}\right)=0
\end{array}
$$

Using Equations (1) and (2) to eliminate $v_{1}$ and $v_{2}$ from Equations (3) and (4) gives

$$
\begin{aligned}
& f_{1}(\theta, \beta)=\cos (\theta) \cdot p_{1}+\sin (\theta) \cdot q_{1}+R_{\text {tool }}=0 \\
& f_{2}(\theta, \beta)=\cos (\beta) \cdot p_{2}+\sin (\beta) \cdot q_{2}-R_{\text {tool }}=0
\end{aligned}
$$

where 


$$
\begin{aligned}
p_{1} & =(T-B) \cdot T_{m}-B_{b} \cdot T_{m} \cdot R_{\mathrm{tool}} \sin (\beta)-B_{m} \cdot T_{m} \cdot R_{\mathrm{tool}} \cos (\beta) \\
q_{1} & =(T-B) \cdot T_{b}-B_{b} \cdot T_{b} \cdot R_{\mathrm{tool}} \sin (\beta)-B_{m} \cdot T_{b} \cdot R_{\mathrm{tool}} \cos (\beta) \\
p_{2} & =(T-B) \cdot B_{m}+T_{b} \cdot B_{m} \cdot R_{\mathrm{tool}} \sin (\theta)+T_{m} \cdot B_{m} \cdot R_{\mathrm{tool}} \cos (\theta) \\
q_{2} & =(T-B) \cdot B_{b}+T_{b} \cdot B_{b} \cdot R_{\mathrm{tool}} \sin (\theta)+T_{m} \cdot B_{b} \cdot R_{\mathrm{tool}} \cos (\theta)
\end{aligned}
$$

The two transcendental equations (5), (6) can be solved for the two unknowns $\theta$ and $\beta$. Subsequently, the two points $v_{1}$ and $v_{2}$ on the tool axis are calculated from the angles $\theta$ and $\beta$ with equations (1) and (2).

\subsection{3-Step optimization}

Bedi et al.'s method uses the boundary curves of a ruled surface as guiding rails. The new algorithm searches for optimum guiding rails that lie anywhere on the ruled surface. Therefore, the above-described method had to be extended to allow for tangency points with different parameter values $u$ and $w$. The points $T(u)$ and $B(u)$ in Equations (1),(2) were replaced by the points $\operatorname{Surf}_{T}(u, w)$ and $\operatorname{Surf}_{B}(u, w)$, where Surf is a ruled surface. At these surface points the Frenet frames were established on the isoparametric curves in $u$ : the tangent $\operatorname{Surf}_{T_{t}}(u, w)$, the normal $\operatorname{Surf}_{T_{m}}(u, w)$ and the binormal $\operatorname{Surf}_{T_{b}}(u, w)$ (similar for the second surface point: $\operatorname{Surf}_{B_{t}}, \operatorname{Surf}_{B_{m}}$ and $\operatorname{Surf}_{B_{b}}$ ).

Including these changes in Bedi et al.'s method allows positioning the cutting tool tangential to any two isoparametric curves (in $u$ ) on the ruled surface. To find the optimum guiding rails, for which the deviation between the designed surface and the ruled surface is minimized, a 3 -step algorithm was developed. Rolling the cylindrical tool along the optimum guiding rails will finally lead to three tangency points at each tool position, two of which are tangencies between the tool and isoparametric curves on the surface, and the third of which is a tangency between the tool and a rule line. To find the optimum rails, the following three steps are conducted, as illustrated in Figure 2.

Step 1: Initialization by solving with Bedi et al.'s method

For a particular $u$ value $\bar{u}$, the first step solves the two transcendental equations (5) and (6) with the Newton Raphson algorithm. From these $\theta, \beta$ values, we get a tool position that places the tool tangent to the points $\operatorname{Surf}_{T}(\bar{u}, 0)$ and $\operatorname{Surf}_{B}(\bar{u}, 1)$. This tool position would have a maximum undercut of the rule line $\operatorname{Surf}(\bar{u}, w)$ at $w=0.5$. Only along the boundary curves of the ruled surface will the machined surface match the designed surface exactly. The resulting distribution of deviation along the rule line between design surface and machined surface is shown in Figure 3 (Step 1).

Step 2: Optimize in direction of rule line (constant parametric $u$ values)

In the second step, both tangency points are moved towards the mid-curve along the rule line at $\bar{u}$. At each step, we adjust the tool position by using Bedi et al.'s method, only this time the two tangency points occur at $\operatorname{Surf}\left(u, \bar{w}_{1}\right)$ and $\operatorname{Surf}\left(u, \bar{w}_{2}\right)$.

The shift of tangency points in this step reduces the initial undercut from Step 1 at the 


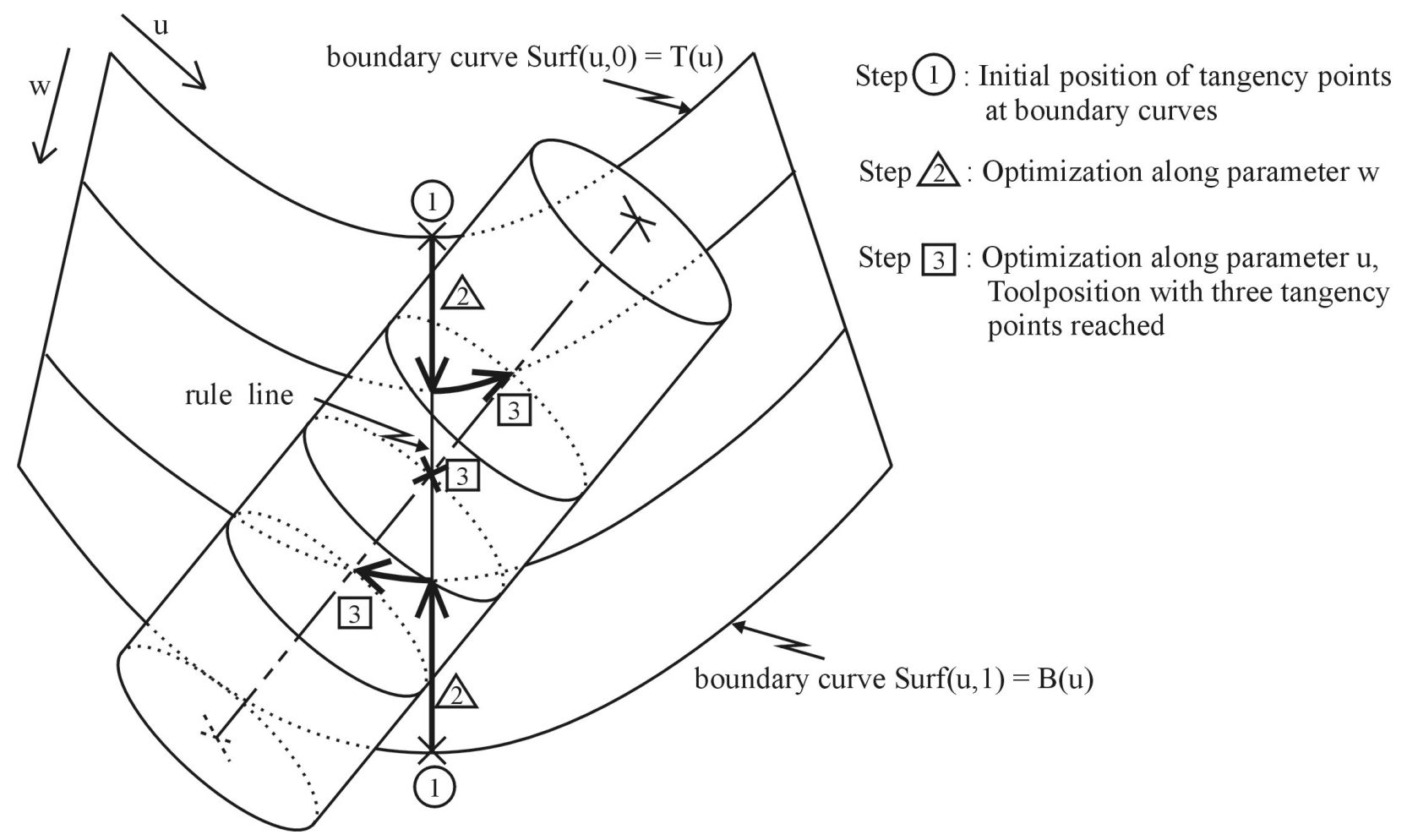

Fig. 2. Schematic drawing of search algorithm

expense of introducing regions of overcut. The maximum overcut occurs at the boundary curves of the surface, while the maximum undercut occurs at the mid-curve. These deviations can be calculated by subtracting the tool radius from the shortest distance between a point on the rule line and the tool axis; positive sign indicates overcut, negative sign undercut. Both tangency points are shifted along the rule line by equal distances from the guiding rails. Keeping the distances equal ensures an even distribution of the deviation, i.e., the magnitude of the overcut deviation is the same at both guiding rails. The values for parameter $w$ are incremented until an optimum position is found at which the error is minimized (i.e., the undercut at the mid-curve will be equal to the maximum overcut at the boundary curves of the surface), making the tool tangent to the isoparametric curves $\operatorname{Surf}\left(u, \bar{w}_{1}\right)$ and $\operatorname{Surf}\left(u, \bar{w}_{2}\right)$ at the points $\operatorname{Surf}_{T}\left(\bar{u}, \bar{w}_{1}\right)$ and $\operatorname{Surf}_{B}\left(\bar{u}, \bar{w}_{2}\right)$ respectively, for some values of $\bar{w}_{1}$ and $\bar{w}_{2}$. The deviation distribution obtained after this step is shown in Figure 3 (Step 2).

Step 3: Optimize in direction of feed (constant parametric $w$ values)

Finally, the two tangency points are shifted in opposite $u$-parameter directions respectively, until the shortest distance between the rule line and the cylinder's axis is equal to $R_{\text {tool }}$, the cylinder's radius. Again, at each step, we adjust the tool position using Bedi et al.'s method. The further the tangency points are moved, the smaller the undercut error will be. Eventually, the deviation switches from undercut to overcut. This point is considered to be optimum, since a third tangency point (to the rule line, rather than to an isoparametric curve) is introduced. The tool is now tangent to the isoparametric curves $\operatorname{Surf}\left(u, \bar{w}_{1}\right)$ and $\operatorname{Surf}\left(u, \bar{w}_{2}\right)$ at the points $\operatorname{Surf}_{T}\left(\bar{u}_{1}, \bar{w}_{1}\right)$ and $\operatorname{Surf}_{B}\left(\bar{u}_{2}, \bar{w}_{2}\right)$ respectively, and it is tangent to the rule line $\operatorname{Surf}(\bar{u}, w)$ at some value $w \in[0,1]$. 


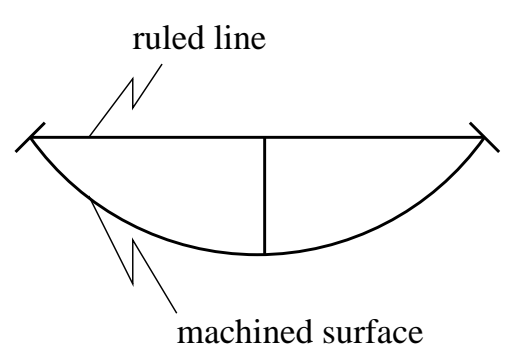

Step 1

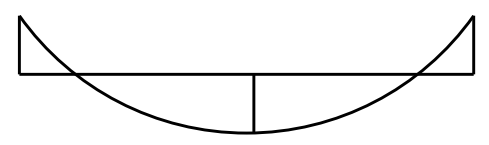

Step 2

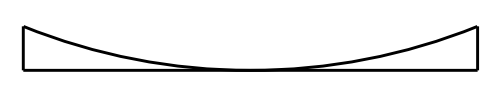

Step 3

Fig. 3. Deviation distribution along rule line after each step

Comparing the one computed tool position to the original desired rule line will show overcuts at the boundary curves of the surface and matching results along the mid-curve of the surface (Figure 3 (Step3)). While one might expect the rule line and the surface to agree at three points, note that after this step the tool will be tangent to the rule line shown in Figure 3 and tangent to two guiding rails at different $u$ values $\bar{u}_{1}$ and $\bar{u}_{2}$.

The key to understanding Step 2 of our algorithm is that for a line that passes through a cylinder at points $P_{1}, P_{2}$, the closest point on the line to the cylinder's axis is $\left(P_{1}+P_{2}\right) / 2[1]$. This is why in Step 1 the maximum undercut of the rule line is at $w=0.5$, and why in Step 2 that by moving $\bar{w}_{1}$ and $\bar{w}_{2}$ by the same increment (but in opposite directions) we know that the maximum undercut of the rule line will remain at $w=0.5$. However, in Step 3, when we adjust the $u$ parameters along the optimized guiding rails, the maximum undercut might not lie at $w=0.5$; thus in Step 3 we optimize over the shortest distance between the rule line and the cylinder's axis.

The deviation calculation from the third step only takes into account this single tool position. Adjacent tool positions will also have to be taken into account when comparing the machined surface to the desired ruled surface. To calculate the true maximum deviation, a comparison between the swept volume [9-11] and the ruled surface is required, as described in the next section.

\section{Simulation}

Swept Volumes is a numerical method that computes grazing curves, which the tool leaves behind on the stock when it moves through 3D-space [11]. Connecting these curves gives the Swept Volume, which is the actual machined surface. We checked the method described in the previous section with a gouge-checking algorithm that uses swept surfaces and was implemented in an existing CAM simulation software developed at the University of Waterloo. The simulation software uses a $z$-map to compute the maximum gouge depth for each tool position and its swept volume to the next tool position.

As a first test (to compare our method to those of Liu [3] and Redonnet,Rubio, and Des- 
Table 1

Control points for Liu surface $[\mathrm{mm}]$

$$
\begin{array}{ccc}
T_{0}=(0 ; 0 ; 33.995) & T_{1}=(11.507 ; 0 ; 33.995) & T_{2}=(23.014 ; 20.2324 ; 33.995) \\
B_{0}=(0 ; 20.429 ; 0) & B_{1}=(11.507 ; 20.429 ; 0) & B_{2}=(23.014 ; 20.429 ; 0)
\end{array}
$$

sein [4]) we use the surface given in Liu's paper as the two rails

$$
T(u)=\left\{\begin{array}{c}
u \\
20.429 \\
0
\end{array} \quad B(u)=\left\{\begin{array}{c}
u \\
0.0382 u^{2} \\
33.995
\end{array}\right.\right.
$$

with $0 \leq u \leq 23.014$ and $0 \leq v \leq 1$. The quadratic Bézier control points for these curves appear in Table 1. In the examples, the surface is machined with a cylindrical tool with radius of $10 \mathrm{~mm}$.

In Table 2 we give the maximum undercut and overcut of four methods on Liu's surface. The values for Liu's and Redonnet-Rubio-Dessein's methods are taken from their papers. This makes a direct comparison a bit more difficult because of the different methods used to compute the errors. In particular, the values for Liu's method was computed by comparing a rule line to the corresponding tool position, while the values we give for Bedi-Mann-Menzel's method and for the method described in this paper were computed as the maximum distance between the rule lines and the swept surface. Redonnet-Rubio-Dessein computed the error by looking at two successive positions rather than the entire swept surface.

For the Bedi-Mann-Menzel method and for the method described in this paper, we used a toolpath comprised of 100 tool positions. In the table, we give the maximum error after each of the three steps of our method. The error appearing in the first column (Step 1) is also the error of the earlier Bedi-Mann-Menzel method. The error of the complete method is that appearing under the Step 3 column. Roughly speaking, our new method has error comparable to Redonnet-Rubio-Dessein's method, which is to be expected since both methods place the tool tangent to the rule line and to two other curves on the ruled surface.

For the method described in this paper, in addition to the maximum error, we also give the maximum angle change between adjacent tool positions (such values were not available for the other two methods).

The test surface in Liu's paper is fairly simple: one rail is a straight line, while the other is a quadratic curve. As a second test, we designed three ruled surfaces to compare the deviations between Bedi et al.'s strategy to the deviations achieved with the optimized method discussed in this paper. One surface has two concave-up curves, the second has one concave-up and one concave-down curve, and the third surface has two concave-down curves. Each ruled surface is comprised of two quadratic Bézier curves; a top curve $T(u)$ and a bottom curve $B(u)$. The control points for the quadratic guiding rails are given in Table 3 and the ruled surfaces 
Table 2

Comparison of methods on Liu surface

\begin{tabular}{r|cc|ccc} 
& & \multicolumn{4}{|c}{ New Method } \\
& Liu & RRD & Step 1 (BMM) & Step 2 & Step 3 \\
\hline Max Undercut [mm] & 0.582 & 0.22 & 2.2393 & 1.1011 & 0.2644 \\
Max Overcut [mm] & 0.585 & 0.22 & 0 & 0.6892 & 0.2114 \\
Max Angle Change & - & - & 0.5984 & 0.5849 & 0.6426
\end{tabular}

Table 3

Control points for ruled surfaces [mm]

\begin{tabular}{r|c|c|c||c|c|c} 
& $T_{0}$ & $T_{1}$ & $T_{2}$ & $B_{0}$ & $B_{1}$ & $B_{2}$ \\
\hline Machined & $(75,15,-5)$ & $(30,30,-5)$ & $(0,60,-5)$ & $(60,0,-45)$ & $(30,30,-45)$ & $(15,75,-45)$ \\
One flipped & $(75,15,-5)$ & $(45,45,-5)$ & $(0,60,-5)$ & $(60,0,-45)$ & $(30,30,-45)$ & $(15,75,-45)$ \\
Two flipped & $(60,0,-5)$ & $(45,45,-5)$ & $(15,75,-5)$ & $(75,15,-45)$ & $(45,45,-45)$ & $(0,60,-45)$
\end{tabular}

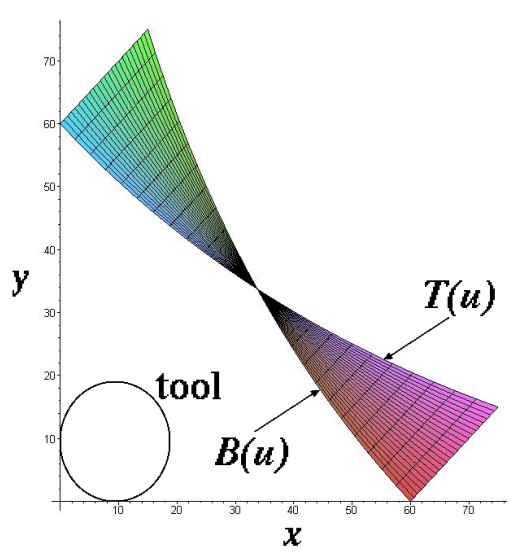

(a) Machined Surface

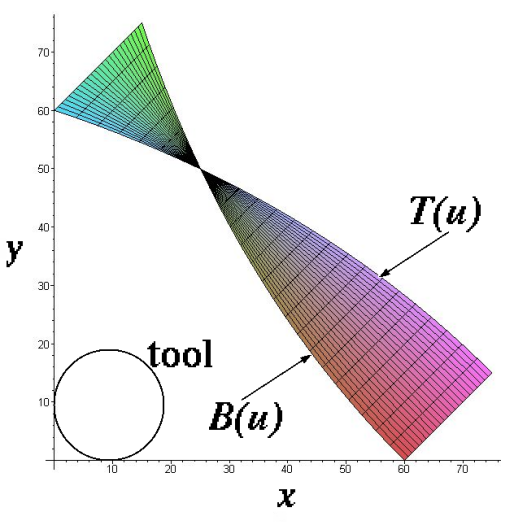

(b) One flipped

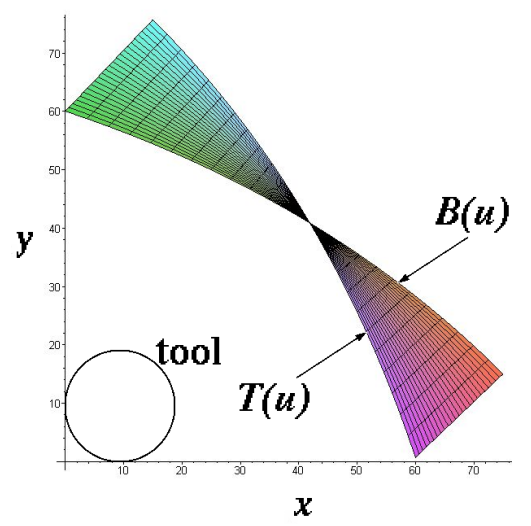

(c) Two flipped

Fig. 4. Ruled Surfaces

are displayed in Figure 4. In these examples, we machined the side of the surface closest to the origin, as indicated by the tool in the figure. Note that in these examples, the pairs of boundary curves lie in planes parallel to the $x y$-plane.

Toolpaths comprised of 100 tool positions for the two positioning algorithms were created using a cutting tool with a radius of $9.5664 \mathrm{~mm}$. The simulations were run on a Pentium III $600 \mathrm{MHz}$ processor. It took 12 seconds to compute the 100 tool positions, where for each tool position, there were an average of 16 Newton-Raphson iterations in step 2 and 20 iterations in step 3 (each Newton-Raphson iteration requires computing a solution to Equations (5),(6)).

Table 4 displays the maximum gouges on the surface for five selected tool positions for both strategies, where the maximum gouges were computed as the maximum distance of the rule line to the swept surface generated by the two methods. In all three of our test surfaces, the 
Table 4

Comparison of methods

\begin{tabular}{c|cc|cc|cc}
\multicolumn{5}{c}{ Maximum deviation ( mm) } \\
& Machined example & One curve flipped & Both curves flipped \\
\hline Position & Bedi & Optimized & Bedi & Optimized & Bedi & Optimized \\
undercut & 0.2899 & 0.0061 & 0.8339 & 0.0099 & 0.2876 & 0.0061 \\
overcut & 0 & 0.0063 & 0 & 0.0169 & 0 & 0.0091 \\
Maximum angle change & between adjacent positions (degrees)
\end{tabular}

\begin{tabular}{|ll|ll|ll}
0.6353 & 0.6225 & 0.9772 & 0.9846 & 0.5727 & 0.5906
\end{tabular}

maximum gouging was reduced by more than $97 \%$. We also computed the overcut of the two methods. Bedi et al.'s method will have 0 overcut, since the segment of interest of the rule line lies entirely inside the tool. The optimization method described in this paper has a nonzero overcut; however, as seen in Table 4, for our examples this overcut is nearly two orders of magnitude smaller than the undercut of the Bedi et al. method. We also machined the surface appearing in Figure 4(a) using both Bedi et al.'s method and the method described in this paper; details on the machining appear in the next section.

This comparison shows that gouges were significantly reduced on the whole surface with the optimized method. The gouging regions produced by both methods were computed with the simulation software and are displayed qualitatively in Figure 5. Note that Figure 3, Step 3 might suggest that there should be no undercut. However, this figure compares the tool to a particular rule line, while the tool is actually tangent to two other curves at different $u$ values. Thus, as indicated in Table 4 , there will be some undercutting.

A few notes on our method as revealed by the simulations are in order. First, note the $w$ parameters $\bar{w}_{1}$ and $\bar{w}_{2}$ give the two isoparametric curves to which the tool is tangent. Initially, $\bar{w}_{1}=0$ and $\bar{w}_{2}=1$, which we adjust in step 2 of our algorithm to equalize the overcut and undercut. The only restrictions we place on these two values is $\bar{w}_{1} \in[0,0.5]$ and $\bar{w}_{2} \in[0.5,1]$. However, in our simulations, the optimization settled on values near 0.14 and 0.86 respectively, and never approached the ends of these intervals.

A second note regards the optimized $u$ parameters and the angle of the tool axis. In step 1 of our algorithm, we use the same $u$ value for both points of tangency. In step 3 of our algorithm, we adjust the two $u$ values in opposite directions to make the tool tangent to two isoparametric lines and tangent to the rule line. A comparison of the tool axis given in the first step (i.e., by Bedi et al.'s method) to our method showed in our examples a change in angle by as much as 5 degrees. However, as shown in the last line of Table 4, the maximum change in the tool axis angle between adjacent tool positions within one method was less than 1 degree.

Finally, note that the optimization method reduces the error between a single tool position and a single rule line at parameter value $u$. Potentially, the tool at one position $u$ might 


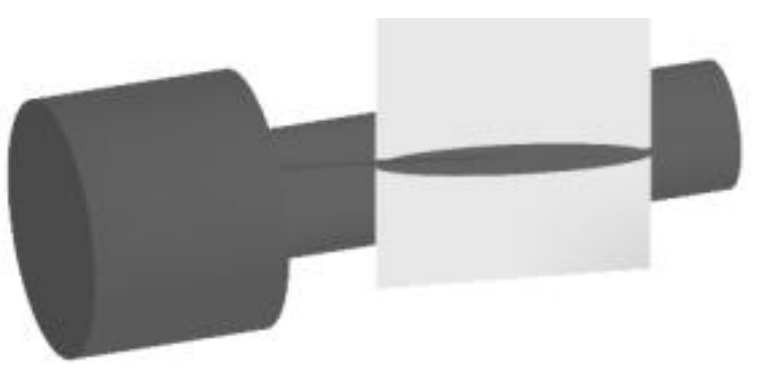

(a) Bedi et al.'s method

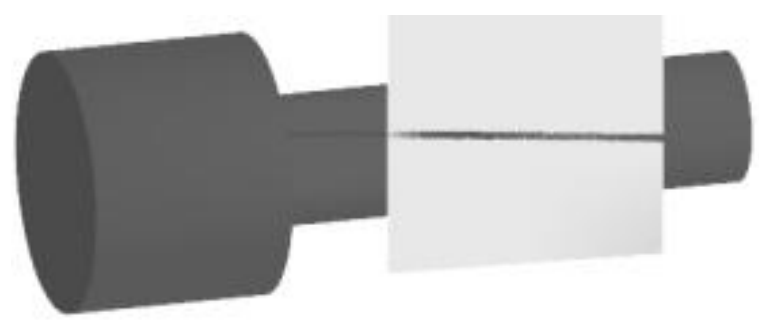

(b) Optimized method

Fig. 5. Graphical simulation of the two positioning strategies

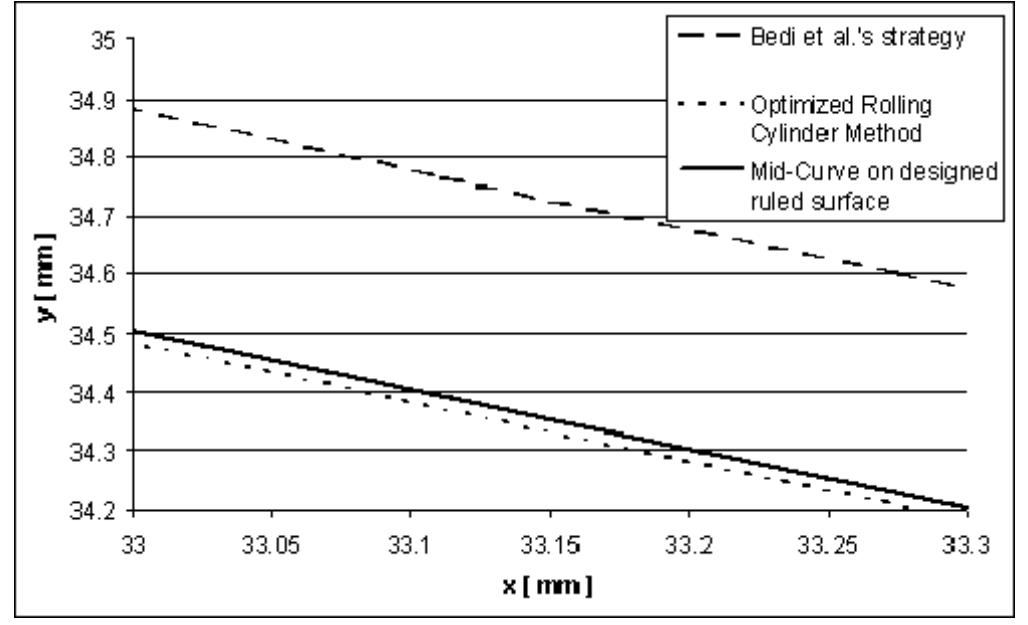

Fig. 6. Comparison of measured data with designed ruled surface $(\mathrm{w}=0.5)$

significantly cut another, nearby rule line at parameter value $u^{\prime}=u+\epsilon$. We did not see this problem in the swept volume analysis of our examples.

\section{Machining Test}

A machining test was conducted to confirm the simulation results. Two parts were flank milled with a flat end cutter on a Deckel Maho 80Pi Hi-Dyn five-axis machine using the two toolpaths described in the simulation section (i.e., one was machined with Bedi et al. method, while the other was machined with the new method described in this paper). Measurements along the mid-curve of the machined surfaces were taken and compared to each other. The deviation of the machined mid-curves from the designed ruled surface is displayed in Figure 6. In this figure, we have graphed a portion of the $w=0.5$ curves; only the $x, y$ values of the curves are shown since all the curves have the same $z$-values because the boundary curves lie in planes parallel to the $x y$-plane. The depicted sections of the mid-curve show that the machining results match closely to the expected simulation results at tool position number 50 (see Table 4). 


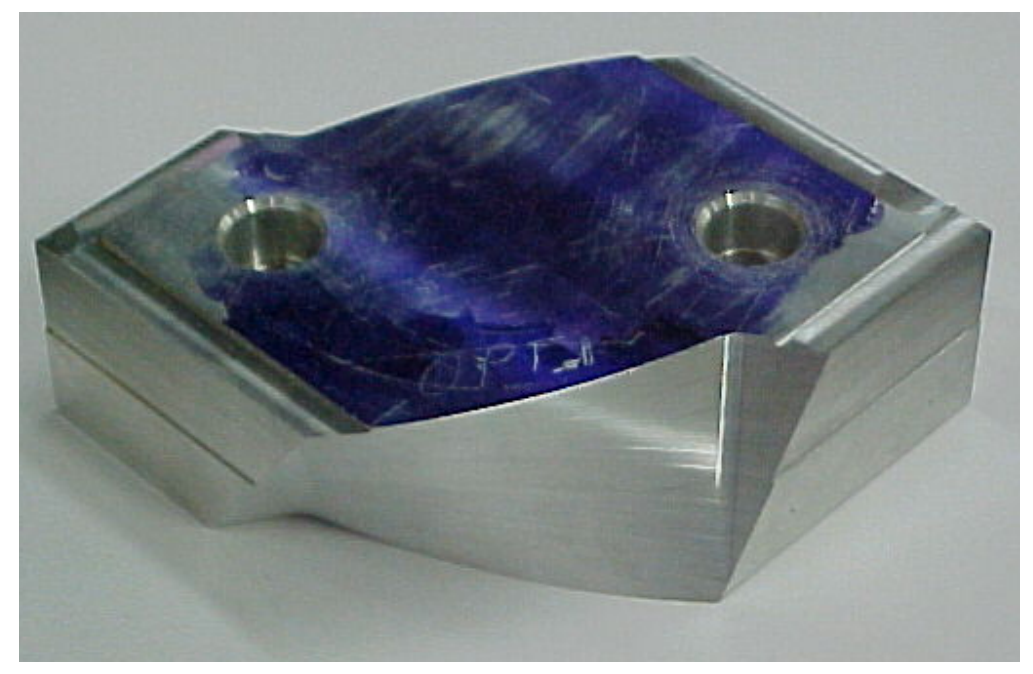

Fig. 7. Machined Part, where $T$ is the top curve and $B$ is the bottom curve.

\section{Conclusions}

In flank milling most research work focuses on machining ruled surfaces. Several positioning strategies have been developed to minimize the deviations between the design surface and the actual machined surface. The main goal of this study was to develop and implement an optimized method for flank milling ruled surfaces. Our method was based on the earlier work of Bedi et al. [1], which gave a method for placing a cylindrical tool tangent to two, fixed guiding rails.

For the optimized method, the guiding rails at which the cutting tool and ruled surface are positioned tangential to each other are no longer restricted to the two boundary curves, but can be located anywhere on the ruled surface. The new algorithm will find the optimum guiding rails for which the deviation is minimized. Placing the tool tangential to these optimum guiding rails leads to three tangency points for each tool position: one point on each optimized guiding rail and the third point on the rule line.

The optimized method reduces the deviation by more than $88 \%$ compared to the strategy proposed by Bedi et al. Furthermore, only two equations need to be solved for numerically, which makes the algorithm computationally inexpensive and robust.

\section{Acknowledgements}

This work was funded in part by NSERC, CFI, and OIT. 


\section{References}

[1] S. Bedi, S. Mann, and C. Menzel. Flank milling with flat end cutters. Computer Aided Design, 35:293-300, 2003.

[2] E. L. J. Bohez, S. D. R. Senadhera, K. Pole, J. R. Duflou, and T. Tar. A geometric modeling and five-axis machining algorithm for centrifugal impellers. Journal of Manufacturing Systems, 16(6):422-463, 1997.

[3] X. W. Liu. Five-axis NC cylindrical milling of sculptured surfaces. Computer-Aided Design, 27(12):887-894, 1995.

[4] J.-M. Redonnet, W. Rubio, and G. Dessein. Side milling of ruled surfaces: Optimum positioning of the milling cutter and calculation of interference. International Journal for Advanced Manufacturing Technology, 14:459-465, 1998.

[5] F. Rehsteiner and H. J. Renker. Collision free five axis milling of twisted ruled surfaces. Annals of CIRP, 42(1):267-271, 1993.

[6] H. K. Tönshoff and N. Rackow. A CAM software prototype for five axis flank milling free-form surfaces. In Proceedings of the Third International Conference on Metal Cutting and High Speed Machining, pages 139-147, June 2001.

[7] C. W. Wu. Arbitrary surface flank milling of fan, compressor, and impeller blades. Journal of Engineering for Gas Turbines and Power, 117:534-539, July 1995.

[8] D. M. Tsay and M. J. Her. Accurate 5-axis machining of twisted ruled surfaces. Journal of Manufacturing Science and Engineering, 123:731-738, 2001.

[9] K. Sheltami, S. Bedi, and F. Ismail. Swept volumes of toroidal cutters using generating curves. International Journal of Machine Tools and Manufacture, 38:855-870, 1998.

[10] D. Roth, S. Bedi, F. Ismail, and S. Mann. Surface swept by a toroidal cutter during 5-axis machining. Computer Aided Design, 33(1):57-63, 2001.

[11] D. J. Roth. Surface swept by a toroidal cutter during 5-axis machining of curved surfaces and its application to gouge checking. Master's thesis, Mechanical Engineering, University of Waterloo, 1999. 